\title{
Effectiveness of fixed-site high-frequency transcutaneous electrical nerve stimulation in chronic pain: a large-scale, observational study
}

This article was published in the following Dove Press journal: Journal of Pain Research

\author{
Xuan Kong \\ Shai N Gozani \\ NeuroMetrix, Inc., Waltham, MA, USA
}

Objective: The objective of this study was to assess the effectiveness of fixed-site high-frequency transcutaneous electrical nerve stimulation (FS-TENS) in a real-world chronic pain sample.

Background: There is a need for nonpharmacological treatment options for chronic pain. FSTENS improved multisite chronic pain in a previous interventional study. Large observational studies are needed to further characterize its effectiveness.

Methods: This retrospective observational cohort study examined changes in chronic pain measures following 60 days of FS-TENS use. The study data were obtained from FS-TENS users who uploaded their device utilization and clinical data to an online database. The primary outcome measures were changes in pain intensity and pain interference with sleep, activity, and mood on an 11-point numerical rating scale. Dose-response associations were evaluated by stratifying subjects into low ( $\leq 30$ days), intermediate (31-56 days), and high ( $\geq 57$ days) utilization subgroups. FS-TENS effectiveness was quantified by baseline to follow-up group differences and a responder analysis ( $\geq 30 \%$ improvement in pain intensity or $\geq 2$-point improvement in pain interference domains).

Results: Utilization and clinical data were collected from 11,900 people using FS-TENS for chronic pain, with 713 device users meeting the inclusion and exclusion criteria. Study subjects were generally older, overweight adults. Subjects reported multisite pain with a mean of 4.8 (standard deviation [SD] 2.5) pain sites. A total of $97.2 \%$ of subjects identified low back and/ or lower extremity pain, and $72.9 \%$ of subjects reported upper body pain. All pain measures exhibited statistically significant group differences from baseline to 60-day follow-up. The largest changes were pain interference with activity $(-0.99 \pm 2.69$ points $)$ and $\operatorname{mood}(-1.02 \pm 2.78$ points $)$. A total of $48.7 \%$ of subjects exhibited a clinically meaningful reduction in pain interference with activity or mood. This proportion increased to $57.1 \%$ for the high utilization subgroup.

Conclusion: FS-TENS is a practical option for treating multisite chronic pain. The greatest impact is on pain interference with activity and mood. FS-TENS utilization and effectiveness exhibit a dose-response association, suggesting that daily use maximizes pain relief.

Keywords: chronic pain, multisite, widespread, transcutaneous electrical nerve stimulation, wearable, pain relief devices

\section{Introduction}

The Functioning and Disability Supplement of the 2012 National Health Interview Survey estimated that 40 million US adults have pain every day or most days and another 87 million have pain on some days. ${ }^{1}$ Many people with chronic pain also have low quality sleep, anxiety, depression, and poor overall health. ${ }^{2}$ The annual economic cost of chronic pain is $\$ 600$ billion in the USA alone. ${ }^{3}$ Prescription opioids are frequently
Correspondence: Shai N Gozani

NeuroMetrix, Inc., 1000 Winter Street, Waltham, MA 0245I, USA

$\mathrm{Tel}+\mathrm{I} 78|3| 42789$

Fax +I 78I 890 I556

Email gozani@neurometrix.com 
used for chronic pain despite concerns about adverse events and addiction. ${ }^{4}$ Opioid alternatives, including nonsteroidal anti-inflammatory drugs and antiepileptics, are also complicated by side effects ${ }^{5,6}$ and potential abuse. ${ }^{7}$ Therefore, there is a need for nonpharmacological treatments for chronic pain.

Transcutaneous electrical nerve stimulation (TENS) is the delivery of electric current across the intact surface of the skin to activate sensory nerve fibers, primarily for pain relief. ${ }^{8}$ TENS is characterized by a number of electrical parameters including the stimulation pulse shape, amplitude, duration, pattern, and frequency. ${ }^{9}$ When evaluated with attention to methodological and technical factors, ${ }^{10,11}$ high frequency $(>50 \mathrm{~Hz}$ ) TENS has been shown to be safe and effective in multiple forms of chronic pain. ${ }^{11-16}$ However, it is likely that the efficacy of TENS in chronic pain has been adversely impacted by under dosing. ${ }^{10,17}$ General purpose TENS (GPTENS) devices are designed to enable stimulation essentially anywhere on the body through individually wired electrodes. This is impractical for multisite chronic pain and complicates TENS use during daily activities and sleep.

A conceptual model for how sensory nerve stimulation leads to pain relief was proposed by Melzack and Wall ${ }^{18}$ in 1965. Their theory stipulates that activation of large diameter sensory nerves (A $\beta$ fibers) closes a "pain gate" in the spinal cord that inhibits the transmission of pain signals carried by nociceptive afferents ( $\mathrm{C}$ and $\mathrm{A} \delta$ fibers) to the brain. In the past 20 years, anatomic pathways and molecular mechanisms that may underlie the pain gate have been identified. Sensory nerve stimulation activates the descending pain inhibition system, primarily the periaqueductal gray (PAG) and rostroventral medial medulla (RVM) located in the midbrain and medulla sections of the brainstem, respectively. ${ }^{19}$ The PAG has neural projections to the RVM, which in turn has diffuse bilateral projections into the spinal cord dorsal horn ${ }^{20,21}$ that inhibit ascending pain signal transmission. ${ }^{19-21}$ Enhanced central pain inhibition may also account for the benefits of sensory nerve stimulation in chronic pain of central nervous system origin. ${ }^{22}$

GP-TENS use is guided by the clinical practice of placing electrodes near the patient's pain. ${ }^{23,24}$ Stimulation directly over the site of pain may be optimal; however, it is not required. Alternative stimulation locations are effective, ${ }^{25-27}$ including proximal to the site of pain, ${ }^{22}$ distal to the site of pain, ${ }^{28}$ on the contralateral limb, ${ }^{29-31}$ within the same dermatomes as the pain, ${ }^{32}$ and to unrelated spinal segments. ${ }^{22,33,34}$ Widespread analgesia is likely related to spinal and supraspinal neural circuits that include activation of central pain inhibition. ${ }^{22,35-38}$ Additional mechanisms may include reduction in sympathetic tone,${ }^{39,40}$ reversal of maladaptive changes in the central nervous system (CNS), ${ }^{41}$ and functional interactions between the cardiovascular and pain regulation systems. ${ }^{42}$ The potential for widespread analgesia suggests an alternative stimulation paradigm called fixed-site high-frequency TENS (FS-TENS), which is designed for a specific location rather than according to the patient's pain distribution. A priori knowledge on the anatomy and neurophysiology of a target site enables the development of wearable analgesic devices that are discreet and optimized for long-term, regular use without disrupting daytime activity or sleep. An FS-TENS device, placed on the upper calf, demonstrated clinically meaningful pain relief in an open-label, interventional study of 88 subjects with multisite chronic pain affecting the low back and lower extremities. ${ }^{43}$

Interventional studies in chronic pain, including randomized clinical trials, are generally conducted in structured settings on narrowly selected, homogeneous subjects. Although these studies can have good internal validity, lack of generalizability limits their application to real-world management of chronic pain. ${ }^{44}$ Large-scale observational studies are needed to determine the effectiveness of chronic pain treatments. There have been a few substantial cross-sectional studies of TENS in chronic pain ${ }^{45,46}$ but no large longitudinal studies that examined changes in chronic pain measures in response to therapy. The present study evaluated the effectiveness of FS-TENS in a large heterogeneous chronic pain sample. The analyses were based on utilization and clinical data uploaded to an online database. The primary objective was to quantify changes in pain intensity and interference with function following 60 days of FS-TENS therapy. A secondary objective was to determine if FS-TENS utilization and pain relief exhibit a dose-response relationship.

\section{Methods \\ Study design}

This retrospective cohort study evaluated changes in chronic pain following 60 days of FS-TENS use. The study data were obtained from FS-TENS users who authorized uploading of their device utilization and clinical data to an online database via a mobile application. The analyses were conducted on an image of the database taken on September 22, 2017. All users of the FS-TENS device who agreed to the storage of their data in the database were eligible for the study. Inclusion criteria were users who provided demographic information and chronic pain characteristics and rated their pain intensity and interference with sleep, activity, and mood at baseline and after 60 days of use. Figure 1 depicts the data collection 


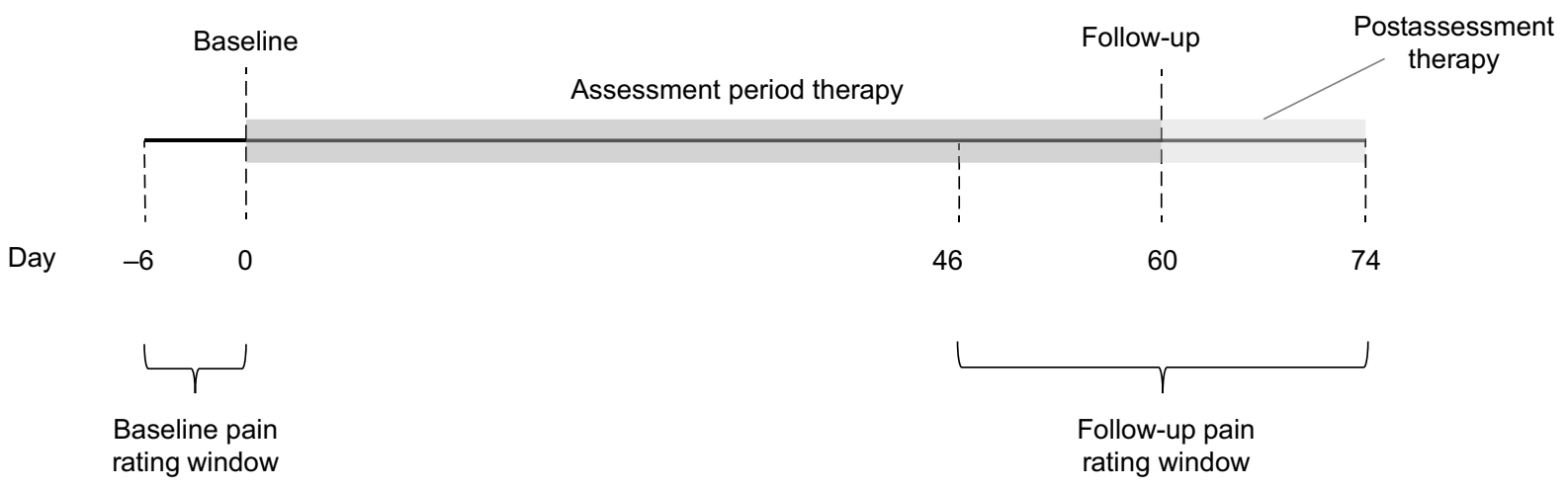

Figure I Schematic illustration of timing of pain ratings and therapy for subjects included in the study cohort.

timeline. A valid baseline pain rating occurred on the first day (day 0 ) of device use or within the prior 6 days. A valid follow-up pain rating occurred between days 46 and 74 (ie, \pm 2 weeks of 60 -day follow-up). If more than one pain rating was available within the baseline or follow-up window, the rating closest to day 0 or 60 , respectively, was used. If more than one pain rating was available on day 0 , the earliest was used. Exclusion criteria were an unspecified pain duration, a pain duration of $<3$ months, an unspecified pain frequency, or a pain frequency less than several times a week.

By authorizing the storage of their data to the online database, device users consented to the manufacturer's privacy policy, which includes use of de-identified data for research purposes. This study was institutional review board exempt because the study authors used an image of the database without personal identifying information (Code of Federal Regulations, Title 45, Department of Health and Human Services, Part 46, Protection of Human Subjects, Section 101(b)(4)). The principles outlined in the World Medical Association Declaration of Helsinki - Ethical Principles for Medical Research Involving Human Subjects - were followed. ${ }^{47}$ The reporting of this study is consistent with the STROBE guidelines. ${ }^{48}$

\section{FS-TENS device}

All FS-TENS users contributing to the database used the same over-the-counter device (Quell ${ }^{\circledR}$; NeuroMetrix, Inc., Waltham, MA, USA).$^{43}$ The device includes a one-channel electrical stimulator, a stretchable band to secure the stimulator to the upper calf, and an electrode. The electrode is an array of hydrogel pads that provide a total stimulation surface area of $60 \mathrm{~cm}^{2}$. When located on the upper calf, the electrode array is circumferential and will stimulate sensory dermatomes S2-L4 independent of rotational placement.
The stimulator generates bipolar, current-regulated pulses with alternating leading phase polarity. Stimulation has zero net current flow (DC) to prevent the development of polar ion concentrations with extended use that may cause skin reactions. The peak output voltage and current are $100 \mathrm{~V}$ and $100 \mathrm{~mA}$, respectively. The pulse duration is either 200 or $280 \mu \mathrm{s}$. The interpulse intervals are random such that the mean stimulation frequency is $80 \mathrm{~Hz}$.

Prior to first use, the device is calibrated to the user's sensation threshold by an algorithm using ascending and descending methods of limits. Subsequent stimulation is controlled automatically. The initial therapeutic level is set so that the pulse charge is $5 \mathrm{~dB}$ above sensation. This level is perceived as "strong but comfortable" ${ }^{46}$ which is the sensation associated with effective high-frequency TENS. ${ }^{14}$ The stimulation intensity is gradually increased by an algorithm to compensate for nerve de-sensitization and to activate deep tissue sensory afferents. ${ }^{49}$ The user may also manually decrease or increase intensity. Each therapy session is 60 minutes, with sessions automatically starting every other hour. The device has a tri-axial accelerometer to monitor body orientation and movement, from which it determines when the user is sleeping using actigraphy methods..$^{50}$ During sleep, the stimulation intensity is reduced to lessen the likelihood of awakening.

The device communicates with a mobile application through Bluetooth ${ }^{\circledR}$. The application is not required for therapeutic use of the device. It provides ancillary benefits including an indication of device status, creation of a demographic and clinical profile, tracking of pain ratings, visualization of device utilization and clinical data, and uploading of data to the online database. This study was based on the online database; therefore, all study subjects used the mobile application. However, the extent of use and impact on compliance and study outcomes were not evaluated. 


\section{FS-TENS database}

The online FS-TENS database includes device utilization, demographics, chronic pain characteristics, daily pain ratings, and objective physiological measurements. All data are electively provided by device users. The origin of the data is either the device (utilization and physiological measurements) or the mobile application (demographics, pain characteristics, and pain ratings). This study evaluated device utilization, demographics, a subset of chronic pain characteristics, and pain ratings. Device utilization included the stimulation intensity measured in milliamps, the number of 1 hour therapy sessions each day, and the number of nights with device use. Demographics included the user's age, gender, height, and weight. Chronic pain characteristics included duration, painful health conditions, anatomic pain distribution, and frequency of pain. The choices for duration were " $\leq 3$ months", " $<1$ year", "1-3 years", "3-10 years", " $>10$ years", and "not sure". Painful health conditions were one or more among 16 medical conditions that are associated with chronic pain. Pain distribution was one or more among 11 sites. Pain was not qualified as unilateral or bilateral because of the potential for contralateral secondary hyperalgesia and allodynia. ${ }^{51}$ Pain frequency was "every day or most days", "several times a week", "several times a month", or "not sure".

Pain intensity and interference were rated on an 11-point numerical rating scale derived from the Brief Pain Inventory - Short Form. ${ }^{52}$ The following four pain domains were assessed: average pain intensity over the past 24 hours, pain interference with sleep over the past 24 hours, pain interference with activity over the past 24 hours, and pain interference with mood over the past 24 hours. There was no schedule or requirement for device users to rate their pain.

\section{Outcome measures and data analysis}

Device utilization, demographics, and pain characteristics were quantified by the mean and standard deviation (SD) if continuous and by frequency counts if categorical. Ninetyfive percent confidence intervals (CIs) for frequency counts were determined using the modified Wald method. Although pain measures defined over a numerical rating scale are ordinal variables, they were treated as continuous variables for statistical analyses. ${ }^{53,54}$ The primary outcome measures were changes in pain intensity and pain interference from baseline to 60-day follow-up. The statistical significance of these changes was determined by the nonparametric Wilcoxon signed-rank test.
Dose-response associations were explored by stratifying subjects into three utilization subgroups based on the number of days they used their device, irrespective of the daily therapy session count. The maximum number of days was 61 . The utilization cutoffs were prospectively set to 50 and $95 \%$ of the 60-day follow-up period. These levels were chosen to represent infrequent use (less than every other day) and daily use. Low utilization was defined as $\leq 30$ days, intermediate utilization was defined as 31-56 days, and high utilization was defined as $\geq 57$ days. Group differences among the three utilization subgroups were evaluated by one-way ANOVA and a two-sample $t$-test for post hoc analyses.

FS-TENS responders were defined for each of the pain domains. A pain intensity responder was a subject with $\geq 30 \%$ improvement from baseline to follow-up. ${ }^{53,55}$ Pain interference responders were subjects with comparable $\geq 2$-point improvement from baseline to follow-up..$^{55}$ When reporting responder rates for a combination of two pain domains (eg, pain interference with activity or mood), subjects with a $\geq 2$-point worsening in either of the same pain domains (eg, pain interference or mood) were excluded to address Type 1 errors due to multiple comparisons. All analyses were performed with Matlab Version R2017a (Mathworks, Natick, MA, USA).

\section{Results}

Utilization and clinical data collected between January 2017 and September 2017 from 11,900 people using FSTENS for chronic pain were evaluated. Out of this data set, baseline and 60-day follow-up pain ratings were available for 713 device users who formed the study cohort and are hereafter referred to as study subjects. The study cohort and the 11,187 excluded FS-TENS users differed with respect to female gender ( 52.3 vs $42.9 \%, P=0.0008$, Pearson chi-squared test), body mass index (BMI) (30.3 \pm 7.0 vs $29.4 \pm 6.6, P=0.0021$, two-group $t$-test), pain duration $>3$ years ( 73.9 vs $62.0 \%, P<0.0001$, Pearson chi-squared test), number of painful health conditions $(3.6 \pm 2.2 \mathrm{vs}$ $2.9 \pm 2.0, P<0.0001$, two-group $t$-test), and number of pain sites ( $4.8 \pm 2.5$ vs $4.0 \pm 2.4, P<0.0001$, two-group $t$-test). Among the excluded FS-TENS users, 2,478 (22\%) users had a baseline pain rating. There were no statistically significant differences between these users and the study subjects with respect to baseline pain intensity or pain interference with sleep, activity, or mood.

Table 1 lists the demographic and pain characteristics for the study cohort and for the subgroups stratified by FS-TENS 
Table I Demographics and pain characteristics of study cohort

\begin{tabular}{|c|c|c|c|c|c|}
\hline \multirow[t]{2}{*}{ Characteristic } & \multirow[t]{2}{*}{ All $(\mathbf{N}=7 \mid 3)$} & \multicolumn{3}{|c|}{ Utilization subgroups } & \multirow[t]{2}{*}{$P$-value } \\
\hline & & Low $(n=148)$ & Intermediate $(n=327)$ & High $(n=238)$ & \\
\hline Female, $\mathrm{n}(\%)$ & $373(52.3)$ & $74(50.0)$ & $179(54.7)$ & $120(50.4)$ & 0.3663 \\
\hline Age (years), mean (SD) & $55.4(13.7)$ & $55.0(14.7)$ & $55.2(13.8)$ & $55.9(\mid 3.1)$ & 0.8028 \\
\hline BMI $\left(\mathrm{kg} / \mathrm{m}^{2}\right)$, mean $(\mathrm{SD})$ & $30.3(7.0)$ & $31.9(8.0)$ & $30.2(7.1)$ & $29.7(6.2)$ & 0.0305 \\
\hline Duration of pain $>3$ years, $n(\%)$ & $527(73.9)$ & $103(69.6)$ & $236(72.2)$ & $188(79.0)$ & 0.0061 \\
\hline Daily pain, n (\%) & 691 (96.9) & $143(96.6)$ & $3 I I(95.1)$ & $237(99.6)$ & 0.0096 \\
\hline Number of pain sites, mean (SD) & $4.8(2.5)$ & $4.8(2.5)$ & $4.8(2.5)$ & $4.9(2.6)$ & 0.7191 \\
\hline Number of painful health conditions, mean (SD) & $3.6(2.2)$ & $3.7(2.4)$ & $3.6(2.3)$ & $3.7(2.2)$ & 0.8998 \\
\hline \multicolumn{6}{|l|}{ Baseline pain, mean (SD) } \\
\hline Average pain & $6.23(1.95)$ & $6.28(2.02)$ & $6.22(1.95)$ & $6.21(1.91)$ & 0.9491 \\
\hline Sleep interference & $5.23(2.94)$ & $5.84(2.84)$ & $5.03(3.01)$ & $5.1 \mathrm{I}(2.87)$ & 0.0151 \\
\hline Activity interference & $6.61(2.43)$ & $6.93(2.50)$ & $6.60(2.43)$ & $6.43(2.35)$ & 0.1458 \\
\hline Mood interference & $6.29(2.63)$ & $6.46(2.67)$ & $6.20(2.67)$ & $6.30(2.55)$ & 0.6039 \\
\hline
\end{tabular}

Notes: Low, therapy on $\leq 30$ days. Intermediate, therapy on 3 I-56 days. High, therapy on $\geq 57$ days. P-value is for one-way ANOVA among the three utilization subgroups. Abbreviation: SD, standard deviation.

utilization. The study subjects were generally older adults, overweight, and equally split among male and female gender. Most subjects reported chronic pain of $>3$ years duration that was characterized by daily pain. More than $90 \%$ of subjects had moderate $(44.2 \%, 4-6$ points on 11-point numerical rating scale) or severe $(47.1 \%, \geq 7$ points on 11-point numerical rating scale) pain at baseline. Subjects reported substantial pain interference with activity and mood, and somewhat less interference with sleep at baseline. As shown in Table 2, the study subjects had extensive pain-related medical histories. Table 3 provides the anatomic distribution of pain sites. Nearly all subjects (97.2\%) indicated low back and/ or lower extremity pain. A substantial proportion (72.9\%) also reported upper body pain (upper extremity, trunk, neck, head, or facial pain).

Stratification of the study cohort into utilization subgroups was not associated with clinically significant differences among demographic and pain characteristics (Table 1). BMI, pain duration, pain frequency, and pain interference with sleep at baseline exhibited statistically significant differences among the three subgroups by oneway ANOVA. The mean pain interference with sleep was higher for the low utilization subgroup when compared with the intermediate $(P=0.0047)$ and high utilization subgroups $(P=0.0149)$.

Table 4 shows FS-TENS usage for the study cohort and for subjects stratified by utilization. On average, study subjects used FS-TENS on $73 \%$ of days and $32 \%$ of nights. The high utilization subgroup used FS-TENS daily. The low utilization subgroup used FS-TENS $\sim 2$ days per week on average. Except for stimulation intensity, there were statistically significant differences among the utilization subgroups. The number of sessions per day increased with
Table 2 Self-reported painful health conditions

\begin{tabular}{ll}
\hline Condition, $\mathbf{n}$ (\%) & $\mathbf{N}=\mathbf{7}$ I3 \\
\hline Musculoskeletal & \\
$\quad$ Arthritis & $444(62.3)$ \\
Fibromyalgia & $182(25.5)$ \\
Spinal & \\
Herniated disc & $217(30.4)$ \\
Spinal stenosis & $205(28.8)$ \\
Neuropathic & \\
Diabetes & $93(13.0)$ \\
Complex regional pain syndrome & $168(23.6)$ \\
Shingles/post herpetic neuralgia & $31(4.3)$ \\
Restless leg syndrome & $143(20.1)$ \\
Multiple sclerosis & $20(2.8)$ \\
Previous injury & \\
Back & $290(40.7)$ \\
Neck & $160(22.4)$ \\
Arm/hand & $155(21.7)$ \\
Leg/foot & $13(1.8)$ \\
Cancer & $4 I(5.8)$ \\
Headaches/migraine & $176(24.7)$ \\
Others & $254(35.6)$ \\
\hline Note:
\end{tabular}

Note: More than one category per participant may apply.

Table 3 Anatomical distribution of pain

\begin{tabular}{ll}
\hline Location, $\mathbf{n}(\%)$ & $\mathbf{N}=\mathbf{7}$ I3 \\
\hline Lower extremity & 641 (89.9) \\
Feet/ankle & $360(50.5)$ \\
Leg (including knees) & $535(75.0)$ \\
Hips & $412(57.8)$ \\
Low back & $575(80.6)$ \\
Trunk (midsection) & $164(23.0)$ \\
Upper extremity & $478(67.0)$ \\
Hands/wrist & $309(43.3)$ \\
Arms & $177(24.8)$ \\
Shoulders & $375(52.6)$ \\
Neck & $359(50.4)$ \\
Head/face & $117(16.4)$ \\
Others & $72(10.1)$ \\
\hline
\end{tabular}

Note: More than one category per participant may apply. 
Table 4 FS-TENS therapy over 60-day assessment period

\begin{tabular}{|c|c|c|c|c|c|}
\hline \multirow[t]{2}{*}{ Parameter, mean (SD) } & \multirow{2}{*}{$\begin{array}{l}\text { All } \\
(N=7 \mid 3)\end{array}$} & \multicolumn{3}{|l|}{ Utilization } & \multirow[t]{2}{*}{$P$-value } \\
\hline & & Low $(n=148)$ & Intermediate $(n=327)$ & High $(n=238)$ & \\
\hline Number of days with $\geq$ I session & $44.5(16.1)$ & $18.4(8.1)$ & $45.2(7.4)$ & $59.7(1.4)$ & 0.0000 \\
\hline Number of nights with $\geq \mathrm{I}$ session & $19.6(19.2)$ & $5.4(6.3)$ & $16.2(14.8)$ & $33.1(21.4)$ & 0.0000 \\
\hline Sessions/day & $6.3(2.6)$ & $4.5(2.0)$ & $5.8(1.9)$ & $8.1(2.7)$ & 0.0000 \\
\hline Total number of sessions & 301 (192) & $88(58)$ & 264 (109) & 485 ( 168$)$ & 0.0000 \\
\hline Mean stimulation level (mA) & $27.1(14.0)$ & $25.9(12.9)$ & $27.2(14.0)$ & $27.4(14.6)$ & 0.5840 \\
\hline
\end{tabular}

Notes: Low, therapy on $\leq 30$ days. Intermediate, therapy on 31-56 days. High, therapy on $\geq 57$ days. P-value is for one-way ANOVA among the three utilization subgroups. Session, I hour continuous stimulation.

Abbreviations: FS-TENS, fixed-site high-frequency transcutaneous electrical nerve stimulation; SD, standard deviation.

Table 5 Changes in pain measures from baseline to 60-day follow-up in FS-TENS users

\begin{tabular}{|c|c|c|c|c|c|}
\hline \multirow[t]{2}{*}{ Pain measure, mean (SD) } & \multirow[t]{2}{*}{ All $(N=7 \mid 3)$} & \multicolumn{3}{|l|}{ Utilization } & \multirow[t]{2}{*}{$P$-value } \\
\hline & & Low $(n=148)$ & Intermediate $(n=327)$ & High $(n=238)$ & \\
\hline Average pain & $-0.37(2.5 \mathrm{I})^{*}$ & $0.23(2.60)$ & $-0.31(2.38)^{*}$ & $-0.82(2.54)^{*}$ & 0.0003 \\
\hline Sleep interference & $-0.31(3.07)^{*}$ & $0.03(3.18)$ & $-0.22(2.97)$ & $-0.65(3.13)^{*}$ & 0.0814 \\
\hline Activity interference & $-0.99(2.69)^{*}$ & $-0.5 \mathrm{I}(2.70)^{*}$ & $-0.94(2.63)^{*}$ & $-1.36(2.74)^{*}$ & 0.0019 \\
\hline Mood interference & $-1.02(2.78)^{*}$ & $-0.22(2.73)$ & $-0.97(2.66)^{*}$ & $-1.58(2.87)^{*}$ & 0.0000 \\
\hline Follow-up (days) & $58.78(5.62)$ & $58.97(7.18)$ & $58.20(5.74)$ & $59.47(4.06)$ & 0.0267 \\
\hline
\end{tabular}

Notes: Low, therapy on $\leq 30$ days. Intermediate, therapy on $31-56$ days. High, therapy on $\geq 57$ days. P-value is for one-way ANOVA among the three utilization subgroups. *Statistical significance of baseline to 60 -day follow-up changes determined by Wilcoxon rank sum test, $P<0.05$.

Abbreviations: FS-TENS, fixed-site high-frequency transcutaneous electrical nerve stimulation; SD, standard deviation.

higher utilization. As a result, total sessions were over fivefold greater for the high utilization subgroup (485) compared to the low utilization subgroup (88).

Table 5 shows baseline to follow-up changes in pain measures. The average assessment period was 58.8 (SD 5.6) days. All pain measures exhibited a statistically significant decrease. The largest changes were observed for pain interference with activity and mood. The responder (ie, $\geq 30 \%$ improvement in pain intensity, $\geq 2$ point improvement in pain interference) rates were $25.7 \%$ (CI 22.6-29.0\%) for pain intensity, $32.4 \%$ (CI 29.1-35.9\%) for pain interference with sleep, $39.4 \%$ (CI 35.9-43.0\%) for pain interference with activity, and $40.1 \%$ (CI $36.6-43.8 \%$ ) for pain interference with mood. A total of $48.7 \%$ of subjects were responders for pain interference with activity or mood. Changes in pain intensity, pain interference with activity and pain interference with mood exhibited statistically significant differences among the utilization subgroups. The responder rates for the high utilization subgroup were $34.5 \%$ (CI $28.7-40.7 \%$ ) for pain intensity, $38.7 \%$ (CI $32.7-45.0 \%$ ) for pain interference with sleep, $46.6 \%$ (CI 40.4-53.0\%) for pain interference with activity, and 51.7\% (CI 45.4-57.9\%) for pain interference with mood. A total of $57.1 \%$ of subjects were responders for pain interference with activity or mood.

\section{Discussion}

This retrospective observational study investigated the effectiveness of FS-TENS in a large heterogeneous chronic pain sample. The study data were obtained from an online database that aggregates utilization and clinical data from individuals with chronic pain using an FS-TENS device. The study cohort consisted of 713 subjects from a total of 11,900 eligible device users. Study subjects and excluded device users differed in several demographic and clinical features. The study cohort was more likely to be female, to have higher BMI, to have more pain sites, to have longer pain duration, and to have more painful health conditions. Given the large sample size, statistically significant differences among demographic and clinical characteristics were expected. However, the differences were small and suggest that the study subjects were comparable to the overall pool of eligible device users with respect to demographic and clinical variables.

Among the 11,187 device users who were not included in the study, 8,709 (78\%) users did not have either a baseline or follow-up pain rating and 2,478 (22\%) users had only a baseline pain rating and no follow-up rating. The small number of device users who provided baseline and follow-up pain ratings was expected. Although device users authorized sharing of their utilization and clinical data, and its use in research, they had no information about relevancy 
of specific data. Device users have the option to track their pain using the mobile application. The benefits of doing so include self-management and facilitation of communication with their physician. However, this feature is elective and is not yet utilized by most device users. Moreover, those who rate their pain do so with variable frequency and timing. The low utilization of pain tracking is likely related to mobile application engagement issues that challenge digital health interventions. ${ }^{56}$

It is reasonable to assume that device users who did not obtain pain relief, who had technical issues (eg, difficulty using the device), or who experienced side effects (eg, skin irritation) during the assessment period were more likely to discontinue therapy than those with positive results. Moreover, device users had a financial incentive to draw an early therapeutic conclusion to comply with a 60 -day product return policy. For this reason, the study cohort may be enriched with FS-TENS responders. The extent of this selection bias cannot be quantified. Therefore, the study findings are generalizable to people with chronic pain completing 2 months of FS-TENS use and not necessarily to all who try FS-TENS. This interpretation is analogous to enriched enrollment randomized withdrawal design, which is common in chronic pain treatment trials ${ }^{57}$ In these studies, a lead in titration phase precedes randomization to active or placebo, such that randomization is performed only on responders.

Key strengths of this study were the real-world setting, large sample size, and heterogeneous distribution of chronic pain. These attributes increase the external validity of the study results. One benefit of evaluating an intervention in a real-world environment is that its use is overlaid on other pharmacological and nonpharmacological therapies. As such, the practical benefits of the intervention were assessed. To our knowledge, this is the largest study investigating longitudinal changes in pain measures in response to TENS therapy. With few exceptions, ${ }^{45,46,58}$ experimental and observational studies of TENS in chronic pain have typically had small sample sizes. ${ }^{10} \mathrm{~A}$ large sample size increases the statistical power to identify relevant outcomes.

Another strength of this study was the quantitative tracking of device utilization. Lack of compliance with selfadministered TENS has been identified as a source of bias that may lead to the underestimation of treatment effects. ${ }^{10,17}$ Poor compliance may include inadequate stimulation intensity ${ }^{10,59}$ and insufficient duration and frequency of stimulation sessions. ${ }^{10}$ The FS-TENS device used in this study addressed these issues through a calibration procedure that establishes a stimulation intensity within the therapeutic window (ie, "strong but comfortable" sensation) and with fixed 1 hour stimulation sessions that automatically start every other hour. In addition, utilization variables including stimulation intensity and daily therapy sessions were electronically monitored.

\section{Study cohort characteristics}

The study cohort reported a mean of 4.8 (SD 2.5) pain sites, which is consistent with recent studies showing that most chronic pain sufferers have widespread pain. ${ }^{1,60,61}$ Multisite pain is associated with substantial comorbidity ${ }^{60,62-65}$ and greater health care utilization. ${ }^{66}$ Nearly all (97\%) study subjects reported low back and/or lower extremity pain. A high number (73\%) of study subjects also reported upper body pain. Anatomically distributed chronic pain represents a practical challenge to treatment with GP-TENS because of the need to place electrodes on multiple body sites. In a recent study, the presence of multisite chronic pain predicted a poor clinical response to GP-TENS. ${ }^{67}$ The present study suggests that FS-TENS may be an effective option for multisite chronic pain.

The study cohort represented a heterogeneous sample of chronic pain. Subjects reported a mean of 3.6 (SD 2.2) painful health conditions. Only $125(17.5 \%)$ subjects reported a single condition, and $461(64.7 \%)$ subjects reported three or more conditions. Arthritis, herniated disc, spinal stenosis, prior back injury, and fibromyalgia were each identified by at least $25 \%$ of the subjects. These disorders are commonly associated with chronic pain and have generally been shown to be responsive to TENS. ${ }^{22,58,68-71}$ The generic use of the term "arthritis" did not enable a more detailed evaluation of the benefits of FS-TENS in specific arthritic conditions. We speculate, based on population prevalence, ${ }^{72}$ that most subjects reporting arthritis had osteoarthritis. More specificity in arthritis categories will be beneficial for future studies. Complex regional pain syndrome (CRPS) was reported with a substantially higher frequency than its population prevalence. ${ }^{73} \mathrm{CRPS}$ is a complex entity that includes inflammatory, sympathetic, neuropathic, immune, and CNS abnormalities ${ }^{74}$ and is difficult to treat. Although few studies of TENS in CRPS have been conducted, there is an indication of potential benefit. ${ }^{75,76}$

The high rate of restless leg syndrome (RLS) reported by the study cohort (20\%) requires comment. Although RLS is classified as a sleep disorder, the sensory symptoms are often described as painful. ${ }^{77,78}$ It is unclear whether RLS was a primary target of FS-TENS therapy or a common comorbidity. RLS is associated with multisite chronic pain, ${ }^{79}$ diabetic neuropathy, ${ }^{80}$ lumbosacral radiculopathy, ${ }^{81}$ muscloskeletal 
pain, ${ }^{82}$ and fibromyalgia. ${ }^{83}$ Periodic leg movements are common in chronic pain ${ }^{84}$ and a biomarker for RLS. There is preliminary evidence that TENS is useful in RLS. ${ }^{85} \mathrm{~A}$ recent open-label pilot study of FS-TENS in RLS yielded encouraging results. ${ }^{86}$ TENS has also been shown to reduce spinal excitability, ${ }^{87}$ which is thought to be a neurophysiological mechanism underlying RLS.

\section{FS-TENS effectiveness}

The Initiative on Methods, Measurement, and Pain Assessment in Clinical Trials (IMMPACT) recommends consideration of six outcome domains when designing studies evaluating chronic pain therapies. These domains are pain, physical function, emotional function, ratings of improvement and satisfaction with treatment, symptoms and adverse events, and subject disposition. ${ }^{88}$ In most clinical studies of chronic pain treatments, pain intensity is the primary outcome measure. However, the most important treatment objective for chronic pain sufferers may not be a reduction in pain intensity. ${ }^{89}$ Enhanced quality of life such as better sleep, greater activity, improved mood, reduced use of pain medications, and fewer side effects may have more practical relevance. Subjects with chronic pain use TENS in various ways in an attempt to improve their quality of life..$^{90}$ This apportioning of the beneficial effects of TENS across multiple pain domains may complicate efforts to demonstrate its efficacy. ${ }^{10}$ In this study, FS-TENS effectiveness was assessed by changes in pain intensity and pain interference with sleep, activity, and mood measured on an 11-point numerical rating scale. The available data did not include a global assessment such as the Patient Global Impression of Change (PGIC) $)^{88}$ or changes in analgesic use.

The results from this study advance our understanding of the impact of FS-TENS on chronic pain. Two months of FS-TENS use resulted in statistically significant reductions in average pain intensity and pain interference with sleep, activity, and mood. The magnitude of the group changes ranged from 0.4 points for pain interference with sleep to 1 point for pain interference with activity and mood. These results reflect real-world use of self-administered FS-TENS as one component of the subject's pain management program. The recorded changes may not reflect all the benefits of FS-TENS in this cohort. Global impression, satisfaction, additional psychological outcomes such as catastrophizing, and pain medication use may have improved but were not represented in the online database. The group changes in pain interference with activity and mood were at the 1-point level that is considered clinically meaningful. ${ }^{55}$ In the responder analysis,
$49 \%$ of subjects achieved a clinically important reduction in pain interference with activity or mood. Although the group changes in pain intensity and pain interference with sleep were modest, some subjects experienced clinically relevant improvement as indicated by the responder analysis. Small group differences may represent substantial clinical benefit to some subjects. ${ }^{91}$

The finding that FS-TENS has a greater influence on pain interference than on pain intensity is consistent with prior studies $^{43,90}$ and relevant to establishing therapeutic expectations and design of future clinical studies. Therapy expectations may influence the outcome of chronic pain interventions, including TENS. Subjects primarily expecting substantial reductions in pain intensity may be disappointed and deem the therapy to be ineffective. By contrast, those focusing on changes in quality of life may consider the therapeutic impact to be substantial. Most randomized and observational studies of TENS therapy in chronic pain have used changes in pain intensity as the primary outcome measure. ${ }^{10}$ This narrow focus may have contributed to low fidelity studies and uncertainty regarding the clinical effectiveness of TENS..$^{90}$

The magnitude and pattern of changes in pain measures in this study are generally similar to an open-label, 60-day interventional study of the same FS-TENS device in 88 subjects with multisite chronic pain. ${ }^{43}$ In that study, $81 \%$ of participants reported that their chronic pain improved based on a 5-point PGIC measure. Among those who reported improvement, $80 \%$ indicated a reduction in concomitant use of pain medications compared to only $12 \%$ in those without improvement. We cannot directly compare these results because the present study did not include a global impression or assessment of pain medication use. As in the current study, the impact on pain interference was greater than on pain intensity; however, the pattern differed. The largest reductions were in pain interference with activity, walking, and sleep, whereas the change in interference with mood was small. The reasons for the differences are unclear but may relate to the distribution of painful health conditions. The prior study ${ }^{43}$ had a higher percentage of subjects reporting diabetes. Painful diabetic neuropathy is associated with poor sleep quality. ${ }^{92}$

We are not aware of other published studies that directly evaluated FS-TENS. However, several studies had relevant methods. Dailey et $\mathrm{al}^{22}$ evaluated the impact of a single TENS treatment on multiple outcome measures in patients with fibromyalgia. The electrodes were applied at either the cervical-thoracic junction or lumbar-sacral junction. The patient's pain threshold was assessed at the following three sites: the site of stimulation, the alternate stimulation site 
(eg, if cervical-thoracic stimulation, then assessed at lumbarsacral junction), and over the anterior tibialis muscle on the leg. The two nonstimulation sites were intended to "assess for widespread effects of TENS outside the site of stimulation". ${ }^{22}$ The study demonstrated increased pain thresholds both at and outside of the site of stimulation. The authors concluded that TENS has widespread effects by decreasing central excitability and activating central pain inhibition mechanisms. Rao et $\mathrm{al}^{25}$ studied the relationship between electrode placement and pain relief in subjects with various forms of chronic pain, including peripheral neuropathy, radiculopathy, and musculoskeletal pain. Electrode locations included the site of pain, proximal to the site of pain, in the same dermatome, at a related trigger or motor point, at a contralateral site, and at a remote site unrelated to the pain. They did not find a correlation between electrode placement and degree of improvement.

\section{FS-TENS dose-response relationship}

A key finding from this study is the positive association between FS-TENS utilization and effectiveness. The baseline to follow-up improvement in all pain measures increased with higher device utilization. The number of subjects meeting the responder definition for pain interference with activity ( 33 vs $47 \%$ ) and mood ( 25 vs $52 \%$ ) increased substantially when comparing the low- and high-utilization subgroups. The proportion of subjects with a clinically important reduction in pain interference with activity or mood increased from 38 to $57 \%$. Similarly, the pain intensity responder rate increased from $18 \%$ in the low-utilization subgroup to $33 \%$ of subjects in the high-utilization subgroup. FS-TENS utilization was determined by the subject rather than controlled. One explanation for this result is the existence of a causal link between FS-TENS dose and effectiveness. Another explanation is that users who benefited from FS-TENS, due to specific or nonspecific mechanisms, used it more often. In either case, it appears that daily, as opposed to intermittent, FS-TENS use provides individuals with chronic pain with the greatest likelihood of meaningful clinical benefit.

The dose-response behavior of TENS has not been studied extensively. Currently published evidence suggests that stimulation intensity directly influences the degree of analgesia in a dose-dependent fashion. ${ }^{59,93}$ Stimulation below the level of sensory perception does not produce analgesia, and the degree of analgesia is correlated to the stimulation intensity. To our knowledge, the present study is the first to show a relationship between the rate of daily TENS use and pain outcomes. Regular users of FS-TENS experienced better pain outcomes than those with less frequent use. Subjects using FS-TENS every day also used their device more during the day than those with lower utilization. As a result, the total number of therapy sessions in the highutilization subgroup was five times greater than that in the low-utilization subgroup. High-frequency TENS is thought to cause transient hypoalgesia through the elevation of enkephalins and the activation of central pain inhibition. ${ }^{19}$ In the present study, $>95 \%$ of subjects reported daily pain. It is possible that those who used their device on an intermittent basis did not experience the same level of pain relief as those who used it daily, due to inadequate dosing. Many TENS studies have used infrequent clinic-based therapy. ${ }^{10}$ Those studies that sent subjects home with a TENS device typically did not have objective monitoring with which to evaluate compliance and utilization. It is probable that some prior TENS studies have been negatively impacted by under dosing. ${ }^{10,17,94}$

\section{FS-TENS mechanism of action}

The principle of widespread analgesia has led to the development of FS-TENS, where the device is designed for a specific location rather than according to the patient's pain distribution. A priori knowledge on the anatomy and neurophysiology of a target site enables the development of wearable analgesic devices that are discreet and optimized for extended use without disrupting daytime activity or sleep. This study re-enforces the effectiveness of FS-TENS in multisite chronic pain. It is unlikely that these results can be explained by a localized model of TENS analgesia. If the FS-TENS device was limited to providing focused pain relief in the legs, consistent with its upper calf location, then we must assume that this effect accounted for the entire observed improvement in pain outcomes. This explanation is unlikely. Leg pain accounted for only $18.4 \%$ (SD 18.0\%) of the total number of pain sites among the study subjects, and $25 \%$ of subjects did not report any leg pain. Moreover, there were no statistically significant differences in baseline to follow-up changes in pain measures among high-utilization subjects with and without leg pain. A more plausible explanation is that a substantial portion of the FSTENS benefit was from analgesia outside of the stimulation area, such as distally in the feet, proximally in the low back region, and possibly extra-segmentally in the upper body. These widespread analgesic effects are likely secondary to segmental mechanisms related to high-frequency stimulation of the S2 to L4 dermatomes and, more broadly, to activation of central pain inhibition. ${ }^{19,22}$ 


\section{Study limitations}

This study had several limitations that need to be considered when interpreting the results. First, the entire study cohort consisted of FS-TENS device users. There was no comparison group that did not receive active FS-TENS therapy. Therefore, the impact of nonspecific effects, such as placebo, cannot be reliably estimated. The observation of a strong dose-response association enhances the likelihood of a causal relationship between FS-TENS and widespread analgesia ${ }^{95}$ but leaves the possibility that measured response is due to nonspecific effects. Controlled studies with randomization to standard and/or sham therapy will be helpful to further understand the mechanisms underlying FS-TENS analgesia. Second, pain outcomes were limited to those available in the database. A global impression of improvement and changes in analgesic use, which showed improvement in a prior study of FS-TENS, ${ }^{43}$ were not available. As a result, the range of potential benefits of FS-TENS in the study cohort could not be determined. Third, it was not possible to quantify the number of users with a baseline pain rating who did not provide a follow-up pain rating due to the lack of FS-TENS effectiveness. Consequently, the impact of selection bias cannot be reliably estimated.

\section{Conclusion}

The results from this study suggest that FS-TENS is an effective option for treating multisite chronic pain in a real-world setting. The most significant impact was a clinically meaningful reduction in pain interference with activity and mood. There were also statistically significant reductions in pain intensity and pain interference with sleep. Pain intensity and pain interference with activity and mood exhibited a doseresponse association. These results further encourage the use of FS-TENS, in the form of wearable devices, for the treatment of chronic pain. The effectiveness of FS-TENS in multisite pain builds support for its widespread analgesic effects, likely arising from the activation of central pain inhibition.

\section{Acknowledgment}

NeuroMetrix, Inc., funded this study.

\section{Author contributions}

SNG was responsible for designing the study and preparing the article. XK was responsible for extracting data from the online database and conducting the statistical analyses. All authors contributed toward data analysis, drafting and critically revising the paper and agree to be accountable for all aspects of the work.

\section{Disclosure}

XK and SNG are employees and shareholders of NeuroMetrix, Inc. The authors report no other conflicts of interest in this work.

\section{References}

1. Nahin RL. Estimates of pain prevalence and severity in adults: United States, 2012. J Pain. 2015;16(8):769-780.

2. Toblin RL, Mack KA, Perveen G, Paulozzi LJ. A population-based survey of chronic pain and its treatment with prescription drugs. Pain. 2011;152(6):1249-1255.

3. Gaskin DJ, Richard P. The economic costs of pain in the United States. J Pain. 2012;13(8):715-724.

4. Turk DC, Wilson HD, Cahana A. Treatment of chronic non-cancer pain. Lancet. 2011;377(9784):2226-2235.

5. van Walsem A, Pandhi S, Nixon RM, Guyot P, Karabis A, Moore RA. Relative benefit-risk comparing diclofenac to other traditional nonsteroidal anti-inflammatory drugs and cyclooxygenase- 2 inhibitors in patients with osteoarthritis or rheumatoid arthritis: a network metaanalysis. Arthritis Res Ther. 2015;17:66.

6. Toth C. Pregabalin: latest safety evidence and clinical implications for the management of neuropathic pain. Ther Adv Drug Saf. 2014;5(1):38-56.

7. Chiappini S, Schifano F. A decade of gabapentinoid misuse: an analysis of the European medicines agency's 'suspected adverse drug reactions' database. CNS Drugs. 2016;30(7):647-654.

8. Johnson MI. Transcutaneous Electrical Nerve Stimulation (TENS): Research to Support Clinical Practice. Oxford: Oxford University Press; 2014.

9. Johnson MI. Transcutaneous electrical nerve stimulation (TENS) and TENS-like devices: do they provide pain relief? Pain Rev. 2001;8:121-158.

10. Bennett MI, Hughes N, Johnson MI. Methodological quality in randomised controlled trials of transcutaneous electric nerve stimulation for pain: low fidelity may explain negative findings. Pain. 2011;152(6):1226-1232.

11. Sluka KA, Bjordal JM, Marchand S, Rakel BA. What makes transcutaneous electrical nerve stimulation work? Making sense of the mixed results in the clinical literature. Phys Ther. 2013;93(10):1397-1402.

12. Johnson M, Martinson M. Efficacy of electrical nerve stimulation for chronic musculoskeletal pain: a meta-analysis of randomized controlled trials. Pain. 2007;130(1-2):157-165.

13. Vance CG, Dailey DL, Rakel BA, Sluka KA. Using TENS for pain control: the state of the evidence. Pain Manag. 2014;4(3):197-209.

14. Johnson MI, Bjordal JM. Transcutaneous electrical nerve stimulation for the management of painful conditions: focus on neuropathic pain. Expert Rev Neurother. 2011;11(5):735-753.

15. Jin DM, Xu Y, Geng DF, Yan TB. Effect of transcutaneous electrical nerve stimulation on symptomatic diabetic peripheral neuropathy: a meta-analysis of randomized controlled trials. Diabetes Res Clin Pract. 2010;89(1):10-15.

16. Cruccu G, Aziz TZ, Garcia-Larrea L, et al. EFNS guidelines on neurostimulation therapy for neuropathic pain. Eur J Neurol. 2007;14(9): 952-970.

17. Pallett EJ, Rentowl P, Johnson MI, Watson PJ. Implementation fidelity of self-administered transcutaneous electrical nerve stimulation (TENS) in patients with chronic back pain: an observational study. Clin J Pain. 2014;30(3):224-231.

18. Melzack R, Wall PD. Pain mechanisms: a new theory. Science. 1965;150(699):971-979.

19. DeSantana JM, Walsh DM, Vance C, Rakel BA, Sluka KA. Effectiveness of transcutaneous electrical nerve stimulation for treatment of hyperalgesia and pain. Curr Rheumatol Rep. 2008;10(6):492-499.

20. Zemlan FP, Behbehani MM, Beckstead RM. Ascending and descending projections from nucleus reticularis magnocellularis and nucleus reticularis gigantocellularis: an autoradiographic and horseradish peroxidase study in the rat. Brain Res. 1984;292(2):207-220. 
21. Antal M, Petko M, Polgar E, Heizmann CW, Storm-Mathisen J. Direct evidence of an extensive GABAergic innervation of the spinal dorsal horn by fibres descending from the rostral ventromedial medulla. Neuroscience. 1996;73(2):509-518.

22. Dailey DL, Rakel BA, Vance CG, et al. Transcutaneous electrical nerve stimulation reduces pain, fatigue and hyperalgesia while restoring central inhibition in primary fibromyalgia. Pain. 2013;154(11):2554-2562.

23. Wall PD, Sweet WH. Temporary abolition of pain in man. Science. 1967;155(3758):108-109.

24. Bergeron-Vezina K, Leonard G. On "what makes transcutaneous electrical nerve stimulation work?.” Sluka KA, Bjordal JM, Marchand S, Rake BA. Phys Ther. 2013;93:1397-1402. Phys Ther. 2013;93(10):1426-1427.

25. Rao VR, Wolf SL, Gersh MR. Examination of electrode placements and stimulating parameters in treating chronic pain with conventional transcutaneous electrical nerve stimulation (TENS). Pain. 1981;11(1):37-47.

26. Brown L, Tabasam G, Bjordal JM, Johnson MI. An investigation into the effect of electrode placement of transcutaneous electrical nerve stimulation (TENS) on experimentally induced ischemic pain in healthy human participants. Clin J Pain. 2007;23(9):735-743.

27. Neto MLP, Maciel LYS, Cruz KML, Filho VJS, Bonjardim LR, DeSantana JM. Does electrode placement influence tens-induced antihyperalgesia in experimental inflammatory pain model? Braz J Phys Ther. 2017;21(2):92-99.

28. Ahmed AR, Ahmed GM, El Gohary A, Shaker E. The immediate effects of transcutaneous electrical nerve stimulation on pain intensity and H-reflex in patients with lumbosacral radiculopathy. Egypt $J$ Neurol Psychiatry Neurosurg. 2010;47:361-366.

29. Kawamura H, Ito K, Yamamoto M, et al. The transcutaneous electrical nerve stimulatoin applied to contralateral limbs for the phantom limb pain. J Phys Ther Sci. 1997;9:71-76.

30. Katz J, France C, Melzack R. An association between phantom limb sensations and stump skin conductance during transcutaneous electrical nerve stimulation (TENS) applied to the contralateral leg: a case study. Pain. 1989;36(3):367-377.

31. Giuffrida O, Simpson L, Halligan PW. Contralateral stimulation, using TENS, of phantom limb pain: two confirmatory cases. Pain Med. 2010;11(1):133-141.

32. Saxena K, Saxena KN, Shokeen S, Taneja B. Comparative evaluation of efficacy of transcutaneous electrical nerve stimulation administered by dermatomal stimulation versus acupuncture points stimulation. North J ISA. 2016;1:29-34.

33. Tsang HH. Diffuse Inhibition of Flexion Reflex by Transcutaneous Electrical Nerve Stimulation (Tens) in Man. [Thesis]. Montreal: McGill University; 1986.

34. Tanaka K, Ikeuchi M, Izumi M, et al. Effects of two different intensities of transcutaneous electrical nerve stimulation on pain thresholds of contralateral muscles in healthy subjects. J Phys Ther Sci. 2015;27(9): 2771-2774.

35. Ainsworth L, Budelier K, Clinesmith M, et al. Transcutaneous electrical nerve stimulation (TENS) reduces chronic hyperalgesia induced by muscle inflammation. Pain. 2006;120(1-2):182-187.

36. Somers DL, Clemente FR. Contralateral high or a combination of highand low-frequency transcutaneous electrical nerve stimulation reduces mechanical allodynia and alters dorsal horn neurotransmitter content in neuropathic rats. J Pain. 2009;10(2):221-229.

37. Chan CW, Tsang H. Inhibition of the human flexion reflex by low intensity, high frequency transcutaneous electrical nerve stimulation (TENS) has a gradual onset and offset. Pain. 1987;28(2):239-253.

38. Dean J, Bowsher D, Johnson MI. The effects of unilateral transcutaneous electrical nerve stimulation of the median nerve on bilateral somatosensory thresholds. Clin Physiol Funct Imaging. 2006;26(5):314-318.

39. Abram SE, Asiddao CB, Reynolds AC. Increased skin temperature during transcutaneous electrical stimulation. Anesth Analg. 1980;59(1):22-25.

40. Kaada B. Vasodilation induced by transcutaneous nerve stimulation in peripheral ischemia (Raynaud's phenomenon and diabetic polyneuropathy). Eur Heart J. 1982;3(4):303-314.
41. Woolf CJ. Central sensitization: implications for the diagnosis and treatment of pain. Pain. 2011;152(3 suppl):S2-S15.

42. Sacco M, Meschi M, Regolisti G, et al. The relationship between blood pressure and pain. J Clin Hypertens. 2013;15(8):600-605.

43. Gozani SN. Fixed-site high-frequency transcutaneous electrical nerve stimulation for treatment of chronic low back and lower extremity pain. J Pain Res. 2016;9:469-479.

44. Rothwell PM. External validity of randomised controlled trials: "to whom do the results of this trial apply?". Lancet. 2005;365(9453):82-93.

45. Fishbain DA, Chabal C, Abbott A, Heine LW, Cutler R. Transcutaneous electrical nerve stimulation (TENS) treatment outcome in long-term users. Clin J Pain. 1996;12(3):201-214.

46. Johnson MI, Ashton CH, Thompson JW. An in-depth study of long-term users of transcutaneous electrical nerve stimulation (TENS). Implications for clinical use of TENS. Pain. 1991;44(3):221-229.

47. Carlson RV, Boyd KM, Webb DJ. The revision of the declaration of Helsinki: past, present and future. $\mathrm{Br} J$ Clin Pharmacol. 2004;57(6):695-713.

48. von Elm E, Altman DG, Egger M, et al. The strengthening the reporting of observational studies in epidemiology (STROBE) statement: guidelines for reporting observational studies. PLoS Med. 2007;4(10):e296.

49. Pantaleao MA, Laurino MF, Gallego NL, et al. Adjusting pulse amplitude during transcutaneous electrical nerve stimulation (TENS) application produces greater hypoalgesia. J Pain. 2011;12(5):581-590.

50. Sadeh A. The role and validity of actigraphy in sleep medicine: an update. Sleep Med Rev. 2011;15(4):259-267.

51. Shenker NG, Haigh RC, Mapp PI, Harris N, Blake DR. Contralateral hyperalgesia and allodynia following intradermal capsaicin injection in man. Rheumatology (Oxford). 2008;47(9):1417-1421.

52. Cleeland CS, Ryan KM. Pain assessment: global use of the Brief Pain Inventory. Ann Acad Med Singapore. 1994;23(2):129-138.

53. Farrar JT, Young JP Jr, LaMoreaux L, Werth JL, Poole RM. Clinical importance of changes in chronic pain intensity measured on an 11-point numerical pain rating scale. Pain. 2001;94(2):149-158.

54. Hartrick CT, Kovan JP, Shapiro S. The numeric rating scale for clinical pain measurement: a ratio measure? Pain Pract. 2003;3(4):310-316.

55. Dworkin RH, Turk DC, Wyrwich KW, et al. Interpreting the clinical importance of treatment outcomes in chronic pain clinical trials: IMMPACT recommendations. J Pain. 2008;9(2):105-121.

56. de Ridder M, Kim J, Jing Y, Khadra M, Nanan R. A systematic review on incentive-driven mobile health technology: as used in diabetes management. J Telemed Telecare. 2017;23(1):26-35.

57. Moore RA, Wiffen PJ, Eccleston C, et al. Systematic review of enriched enrolment, randomised withdrawal trial designs in chronic pain: a new framework for design and reporting. Pain. 2015;156(8):1382-1395.

58. Buchmuller A, Navez M, Milletre-Bernardin M, et al. Value of TENS for relief of chronic low back pain with or without radicular pain. Eur J Pain. 2012;16(5):656-665.

59. Moran F, Leonard T, Hawthorne S, et al. Hypoalgesia in response to transcutaneous electrical nerve stimulation (TENS) depends on stimulation intensity. J Pain. 2011;12(8):929-935.

60. Carnes D, Parsons S, Ashby D, et al. Chronic musculoskeletal pain rarely presents in a single body site: results from a UK population study. Rheumatology (Oxford). 2007;46(7):1168-1170.

61. Peat G, Thomas E, Wilkie R, Croft P. Multiple joint pain and lower extremity disability in middle and old age. Disabil Rehabil. 2006;28(24):1543-1549.

62. Coggon D, Ntani G, Palmer KT, et al. Patterns of multisite pain and associations with risk factors. Pain. 2013;154(9):1769-1777.

63. Dragioti E, Larsson B, Bernfort L, Levin LA, Gerdle B. A cross-sectional study of factors associated with the number of anatomical pain sites in an actual elderly general population: results from the PainS65+ cohort. J Pain Res. 2017;10:2009-2019.

64. Gureje O, Von Korff M, Kola L, et al. The relation between multiple pains and mental disorders: results from the World Mental Health Surveys. Pain. 2008;135(1-2):82-91 
65. Eggermont LH, Bean JF, Guralnik JM, Leveille SG. Comparing pain severity versus pain location in the MOBILIZE Boston study: chronic pain and lower extremity function. J Gerontol A Biol Sci Med Sci. 2009;64(7):763-770.

66. de Fernandes RC, Burdorf A. Associations of multisite pain with healthcare utilization, sickness absence and restrictions at work. Int Arch Occup Environ Health. 2016;89(7):1039-1046.

67. Koke AJ, Smeets RJ, Perez RS, et al. Can we "predict" long-term outcome for ambulatory transcutaneous electrical nerve stimulation in patients with chronic pain? Pain Pract. 2015;15(3):256-264.

68. Carbonario F, Matsutani LA, Yuan SL, Marques AP. Effectiveness of high-frequency transcutaneous electrical nerve stimulation at tender points as adjuvant therapy for patients with fibromyalgia. Eur J Phys Rehabil Med. 2013;49(2):197-204.

69. Law PP, Cheing GL. Optimal stimulation frequency of transcutaneous electrical nerve stimulation on people with knee osteoarthritis. J Rehabil Med. 2004;36(5):220-225.

70. Rutjes AW, Nuesch E, Sterchi R, et al. Transcutaneous electrostimulation for osteoarthritis of the knee. Cochrane Database Syst Rev. 2009;(4):CD002823.

71. Osiri M, Welch V, Brosseau L, et al. Transcutaneous electrical nerve stimulation for knee osteoarthritis. Cochrane Database Syst Rev. 2000;(4):CD002823.

72. Plotnikoff R, Karunamuni N, Lytvyak E, et al. Osteoarthritis prevalence and modifiable factors: a population study. BMC Public Health. 2015;15:1195.

73. Sandroni P, Benrud-Larson LM, McClelland RL, Low PA. Complex regional pain syndrome type I: incidence and prevalence in Olmsted county, a population-based study. Pain. 2003;103(1-2):199-207.

74. Goebel A. Complex regional pain syndrome in adults. Rheumatology (Oxford). 2011;50(10):1739-1750.

75. Somers DL, Clemente FR. Transcutaneous electrical nerve stimulation for the management of neuropathic pain: the effects of frequency and electrode position on prevention of allodynia in a rat model of complex regional pain syndrome type II. Phys Ther. 2006;86(5):698-709.

76. Bilgili A, Cakir T, Dogan SK, Ercalik T, Filiz MB, Toraman F. The effectiveness of transcutaneous electrical nerve stimulation in the management of patients with complex regional pain syndrome: a randomized, double-blinded, placebo-controlled prospective study. J Back Musculoskelet Rehabil. 2016;29(4):661-671.

77. Allen RP, Walters AS, Montplaisir J, et al. Restless legs syndrome prevalence and impact: REST general population study. Arch Intern Med. 2005;165(11):1286-1292.

78. Cho YW, Song ML, Earley CJ, Allen RP. Prevalence and clinical characteristics of patients with restless legs syndrome with painful symptoms. Sleep Med. 2015;16(6):775-778.

79. Stehlik R, Ulfberg J, Hedner J, Grote L. High prevalence of restless legs syndrome among women with multi-site pain: a population-based study in Dalarna, Sweden. Eur J Pain. 2014;18(10):1402-1409.
80. Lopes LA, Lins Cde M, Adeodato VG, et al. Restless legs syndrome and quality of sleep in type 2 diabetes. Diabetes Care. 2005;28(11): 2633-2636.

81. Kocabicak E, Terzi M, Akpinar K, Paksoy K, Cebeci I, Iyigun O. Restless leg syndrome and sleep quality in lumbar radiculopathy patients. Behav Neurol. 2014;2014:245358.

82. Hoogwout SJ, Paananen MV, Smith AJ, et al. Musculoskeletal pain is associated with restless legs syndrome in young adults. BMC Musculoskelet Disord. 2015;16:294.

83. Viola-Saltzman M, Watson NF, Bogart A, Goldberg J, Buchwald D. High prevalence of restless legs syndrome among patients with fibromyalgia: a controlled cross-sectional study. J Clin Sleep Med. 2010;6(5):423-427.

84. Okura K, Lavigne GJ, Huynh N, Manzini C, Fillipini D, Montplaisir JY. Comparison of sleep variables between chronic widespread musculoskeletal pain, insomnia, periodic leg movements syndrome and control subjects in a clinical sleep medicine practice. Sleep Med. 2008;9(4):352-361.

85. Kovacevic-Ristanovic R, Cartwright RD, Lloyd S. Nonpharmacologic treatment of periodic leg movements in sleep. Arch Phys Med Rehabil. 1991;72(6):385-389.

86. Winkelman JW, Mei LA, Platt S, Schoerning L. Pilot open-label trial of transcutaneous electrical nerve stimulation (TENS) below the knee for the treatment of restless legs syndrome (RLS). Sleep. 2016;39(Abstract suppl).

87. Joodaki MR, Olyaei GR, Bagheri H. The effects of electrical nerve stimulation of the lower extremity on H-reflex and F-wave parameters. Electromyogr Clin Neurophysiol. 2001;41(1):23-28.

88. Dworkin RH, Turk DC, Farrar JT, et al. Core outcome measures for chronic pain clinical trials: IMMPACT recommendations. Pain. 2005;113(1-2):9-19.

89. Sullivan MD, Ballantyne JC. Must we reduce pain intensity to treat chronic pain? Pain. 2016;157(1):65-69.

90. Gladwell PW, Badlan K, Cramp F, Palmer S. Direct and indirect benefits reported by users of transcutaneous electrical nerve stimulation for chronic musculoskeletal pain: qualitative exploration using patient interviews. Phys Ther. 2015;95(11):1518-1528.

91. Dworkin RH, Turk DC, McDermott MP, et al. Interpreting the clinical importance of group differences in chronic pain clinical trials: IMMPACT recommendations. Pain. 2009;146(3):238-244.

92. Zelman DC, Brandenburg NA, Gore M. Sleep impairment in patients with painful diabetic peripheral neuropathy. Clin J Pain. 2006;22(8): 681-685.

93. Claydon LS, Chesterton LS, Barlas P, Sim J. Dose-specific effects of transcutaneous electrical nerve stimulation (TENS) on experimental pain: a systematic review. Clin J Pain. 2011;27(7):635-647.

94. Bjordal JM. Time for a paradigm shift in pain treatment: reassessing transcutaneous electrical nerve stimulation (TENS). Pain. 2011;152(6):1213-1214.

95. Hill AB. The environment and disease: association or causation? Proc R Soc Med. 1965;58:295-300.
Journal of Pain Research

\section{Publish your work in this journal}

The Journal of Pain Research is an international, peer reviewed, open access, online journal that welcomes laboratory and clinical findings in the fields of pain research and the prevention and management of pain. Original research, reviews, symposium reports, hypothesis formation and commentaries are all considered for publication.

\section{Dovepress}

The manuscript management system is completely online and includes a very quick and fair peer-review system, which is all easy to use. Visit http://www.dovepress.com/testimonials.php to read real quotes from published authors. 